\title{
CORRESPONDENCE
}

\section{INDUCED ELEVATION OF INTRA-OCULAR PRESSURE AND THE VISUAL FIELD}

\section{To the Editorial Committee of the British Journal of Ophthalmology}

Sirs,-In the May, 1964, issue of the British Journal of Ophthalmology (vol. 48, p. 237), J. C. Tsamparlakis has published the results of his investigations on the effect of transient induced elevation of intra-ocular pressure on the visual field. The conclusions reached by the author, who has suggested an ingenious apparatus to exert a graduated pressure on the eye, closely correspond to those presented by me at the XVIth International Ophthalmological Congress, London, 1950 (Acta, p. 895). I concluded, in effect, that the study of the blind-spot during and after dynamometric compression of the eyeball, owing to its enlargement in early glaucomatous eyes, had to be considered as another valuable test for the early diagnosis of chronic glaucoma. The value of this test has been stressed again in a paper published by me in the Archives of Ophthalmology (1953, vol. 49, p. 491), and independently from me, by Suda (see Suda and others, Acta Soc. ophthal. jap., 1962, vol. 66, p. 1225, and previous papers).

These previous researches have been completely ignored by Dr. Tsamparlakis. Similar results can be obtained also by putting the patient in anoxia.

$$
\begin{aligned}
& \text { Yours faithfully, } \\
& \text { G. B. BIETTI }
\end{aligned}
$$

Director,

Clinica OCulistica dell'Università di Roma,

ITALIA.

December, 1964.

\section{BOOK REVIEWS}

Toxoplasmosis. Argumenta et documenta ophthalmologica. By H. REMKY. 1962. Pp. 154, 93 figs, refs. Lehmann, Munich. (DM 54.)

This monograph describes the results of a survey of 617 patients with endogenous uveitis, with particular emphasis on the role of toxoplasmosis in the aetiology. The literature is briefly reviewed and the method of determining the relative levels of antibody in aqueous and serum described. A comparison of dye-test levels in the aqueous and serum was made in 391 patients and the results of this investigation, correlated with the clinical description of the cases, form the main part of the book. The cases diagnosed as congenital toxoplasmosis presented the classical picture of a focal chorio-retinitis, but the acquired disease showed a wider variety of fundus appearances and included eight cases of central serous retinopathy and one of bilateral cyclitis. The author feels that in the majority of the cases the initial infection was acquired post-natally and that in six there were also extra-ocular manifestations of infection with toxoplasmosis.

Although it is probable that further experience will show that the interpretation of comparative estimations of antibody in aqueous and serum is more complicated than at present appears, this work is a valuable contribution to our knowledge of toxoplasmic uveitis.

The arrangement of the book is unusual in that the main text is presented in four languagesGerman, English, Spanish, and French. The explanatory notes to the large number of excellent fundus photographs are also in these four languages. The book is clearly printed on good paper, and although the duplication of the subject matter adds to the bulk, the translations will be appreciated by the many to whom the German text would have posed severe linguistic difficulties. 